\title{
Compositional universality in the $N$-dimensional ball
}

\author{
L. Bernal-González, A. Bonilla, M. C. Calderón-Moreno
}

Received: November 29, 2005

Summary: It is proved in this note that a sequence of automorphisms on the $N$-dimensional unit ball acts properly discontinuously if and only if its corresponding sequence of composition operators is universal on the Hardy space of such ball, and if and only if there exists a dense linear manifold of universal functions. Our result completes earlier ones by several authors.

\section{Introduction and notation}

Throughout this paper, we will denote by $\mathbb{N}, \mathbb{C}, \mathbb{D}$ the set of positive integers, the complex plane and the open unit disk $\{z \in \mathbb{C}:|z|<1\}$, respectively. The boundary of $\mathbb{D}$ is the unit circle $\partial \mathbb{D}=\{z \in \mathbb{C}:|z|=1\}$. If $N \in \mathbb{N}$ and $G \subset \mathbb{C}^{N}$ is an open subset, then $H(G)$ stands for the Fréchet space of holomorphic functions on $G$ endowed with the topology of uniform convergence on compact subsets. A domain $G \subset \mathbb{C}^{N}$ is a nonempty connected open subset of $\mathbb{C}^{N}$. A domain $G \subset \mathbb{C}$ is said to be simply connected whenever its complement with respect to the extended complex plane is connected. If $1 \leq p<\infty$, the Hardy space $H^{p}(\mathbb{D})$ is defined as

$$
H^{p}(\mathbb{D})=\left\{f \in H(\mathbb{D}):\|f\|_{p}<\infty\right\},
$$

where $\|f\|_{p}=\sup _{0<r<1}\left(\frac{1}{2 \pi} \int_{0}^{2 \pi}\left|f\left(r^{i \theta}\right)\right|^{p} d \theta\right)^{1 / p}$. Then $H^{p}(\mathbb{D})$ becomes a Banach space if it is endowed with this norm. See [10] for an extensive study of Hardy spaces.

The group $\operatorname{Aut}(\mathbb{D})$ of automorphisms of $\mathbb{D}$ is the set of Möbius transformations $\left\{\sigma_{a, k}:|a|<1=|k|\right\}$, where $\sigma_{a, k}(z)=k \cdot \frac{z-a}{1-\bar{a} z}$. In 1941, W. Seidel and J. L. Walsh [16] established the existence of a function $f \in H(\mathbb{D})$ such that, given a simply connected domain $G \subset \mathbb{D}$ and a function $g \in H(G)$, there is a sequence $\left\{a_{n}\right\}_{1}^{\infty} \subset \mathbb{D}$ depending on $g$ such that $f \circ \sigma_{a_{n}, 1} \rightarrow g(n \rightarrow \infty)$ in $H(G)$. This result is in turn a non-Euclidean version of Birkhoff's theorem about density of translates of certain entire functions [3]. Recall

AMS 2000 subject classifications: Primary: 47B33; Secondary: 32A35, 47A16

Key words and phrases: Hardy space, Seidel-Walsh theorem, composition operator, $N$-dimensional ball, algebraically generic universality 
that if $G$ is a domain in $\mathbb{C}^{N}$ then it is said that the action of a sequence $\left\{S_{n}\right\}_{1}^{\infty} \subset \operatorname{Aut}(G)$ is properly discontinuous if and only if, given a compact subset $K \subset G$, there exists $m=m(K) \in \mathbb{N}$ such that $K \cap S_{m}(K)=\emptyset$. In 1995, A. Montes-Rodríguez and the first author [2] extended Seidel-Walsh's theorem by showing that if $\left\{S_{n}=\sigma_{a_{n}, k_{n}}: n \in \mathbb{N}\right\} \subset$ Aut $(\mathbb{D})$, then the set $\left\{f \in H(\mathbb{D}):\left\{f \circ S_{n}\right\}\right.$ is dense in $\left.H(\mathbb{D})\right\}$ is not empty if and only if it is residual if and only if $\sup _{n \in \mathbb{N}}\left|a_{n}\right|=1$ if and only if the action of $\left\{S_{n}\right\}_{1}^{\infty}$ is properly discontinuous on $\mathbb{D}$. In particular, if $\varphi=\sigma_{a, k}\left(|a|<1, k={ }^{i \theta}\right)$ and $S_{n}=\varphi \circ \cdots \circ \varphi(n$ times), then the set $\left\{f \in H(\mathbb{D}):\left\{f \circ S_{n}\right\}\right.$ is dense in $\left.H(\mathbb{D})\right\}$ is not empty if and only if it is residual if and only if $\varphi$ has no fixed point in $\mathbb{D}$ if and only if $|\sin (\theta / 2)| \leq|a|$.

If $\varphi$ is a holomorphic selfmapping on $\mathbb{D}$, then the composition mapping $C_{\varphi}: f \in$ $H^{p}(\mathbb{D}) \mapsto f \circ \varphi \in H^{p}(\mathbb{D})$ is a well-defined linear operator on $H^{p}(\mathbb{D})$ (see $[19$, pages 220-221]). In [5, Theorem 2.3 and Proposition 0.1] (see also [17, Chapter 7]) Bourdon and Shapiro showed that if $\varphi \in \operatorname{Aut}(\mathbb{D})$ and $S_{n}=\varphi \circ \cdots \circ \varphi$ ( $n$ times), then the set $\mathcal{U}=\left\{f \in H^{2}(\mathbb{D}):\left\{f \circ S_{n}\right\}\right.$ is dense in $\left.H^{2}(\mathbb{D})\right\}$ is residual in $H^{2}(\mathbb{D})$ if and only if it is not empty if and only if $\varphi$ has no fixed point in $\mathbb{D}$. A key idea of the proof is the fact that the sequence of iterates converges in $H(\mathbb{D})$ to a point $\gamma \in \partial \mathbb{D}$, called the "Denjoy-Wolff point": see [6] or [17, page 78], for instance.

For $N \in \mathbb{N}$, denote by $\mathbb{B}_{N}$ the unit ball in $\mathbb{C}^{N}$, that is, $\mathbb{B}_{N}=\left\{z=\left(z_{1}, \ldots, z_{N}\right) \in \mathbb{C}^{N}\right.$ : $\left.\sum_{j=1}^{N}\left|z_{j}\right|^{2}<1\right\}$. If $z=\left(z_{1}, \ldots, z_{n}\right)$ and $w=\left(w_{1}, \ldots, w_{N}\right)$ then $|z|=\left(\sum_{j=1}^{N}\left|z_{j}\right|^{2}\right)^{1 / 2}$ and $\left\langle z, w>\right.$ will stand for the expression $<z, w>=\sum_{j=1}^{N} z_{j} \overline{w_{j}}$. If $a \in \mathbb{B}_{N}$, denote

$$
\varphi_{a}(z)=\frac{a-P_{a}(z)-\left(1-|a|^{2}\right)^{1 / 2} Q_{a}(z)}{1-<z, a>},
$$

where $P_{a}$ is the projection onto the space $[a]$ spanned by $a$, i.e., $P_{a}(z)=\frac{<z, a>}{|a|^{2}} a$, and $Q_{a}=I-P_{a}$, projection onto the orthogonal complement of $[a]$. Note that $\varphi_{a} \in H\left(\overline{\mathbb{B}_{N}}\right)$ $\left(\overline{\mathbb{B}_{N}}:=\right.$ the closed unit ball) and that $\varphi$ interchanges the values $a, 0$. It is known (see, for instance, [15, Chapter 2] or [8, Theorem 2.72]) that if $\varphi \in \operatorname{Aut}\left(\mathbb{B}_{N}\right)$ then there exist a unitary transformation $M$ of $\mathbb{C}^{N}$ (i.e., a linear operator that preserves inner products) and a point $a \in \mathbb{B}_{N}$ such that $\varphi=M \circ \varphi_{a}$ (in fact, $a=\varphi^{-1}(0)$ ). In [14], F. LeónSaavedra proves that if $\left\{S_{n}\right\}_{1}^{\infty} \subset \operatorname{Aut}\left(\mathbb{B}_{N}\right)$, then there exists $f \in H\left(\mathbb{B}_{N}\right)$ such that $\left\{f \circ S_{n}: n \in \mathbb{N}\right\}$ is dense in $H\left(\mathbb{B}_{N}\right)$ if and only if the action of $\left\{S_{n}\right\}_{1}^{\infty}$ is properly discontinuous on $\mathbb{B}_{N}\left[14\right.$, Theorem 5] if and only if $\limsup _{n \rightarrow \infty}\left|\varphi_{n}^{-1}(0)\right|=1[14$, Proposition 2], see also [7, Theorem 3]. He also provides an analogous result for the unit polydisk $\mathbb{D}_{N}=\left\{z=\left(z_{1}, \ldots, z_{N}\right) \in \mathbb{C}^{N}:\left|z_{j}\right|<1 \quad(j=1, \ldots, N)\right\}$. Note that density in $H\left(\mathbb{B}_{N}\right)$ does not imply density in $H^{p}\left(\mathbb{B}_{N}\right)$ (see definition and some properties in Section 2 below), because convergence here is stronger than convergence in $H\left(\mathbb{B}_{N}\right)$. In 2001, Xiaoman, Guangfu and Kunyu [18, Theorems 2-3] have shown-for the case $p=2$ that if $\varphi \in \operatorname{Aut}\left(\mathbb{B}_{N}\right)$ and $\varphi$ has no fixed point in $\mathbb{B}_{N}$ then there exists $f \in H^{2}\left(\mathbb{B}_{N}\right)$ such that the set $\left\{f \circ \varphi^{n}: n \in \mathbb{N}\right\}$ is dense in $H^{2}\left(\mathbb{B}_{N}\right)$, where $\varphi^{n}=\varphi \circ \cdots \circ \varphi(n$ fold). On the other hand, the second and third authors proved in [4, Theorem 3.1] that if 
$\left\{S_{n}\right\}_{1}^{\infty} \subset \operatorname{Aut}\left(\mathbb{D}_{N}\right)$, then there exists $f \in H^{p}\left(\mathbb{D}_{N}\right)$ such that $\left\{f \circ S_{n}: n \in \mathbb{N}\right\}$ is dense in $H^{p}\left(\mathbb{D}_{N}\right)$ if and only if the action of $\left\{S_{n}\right\}_{1}^{\infty}$ is properly discontinuous on $\mathbb{B}_{N}$. Note that this does not imply a corresponding result on $H^{p}\left(\mathbb{B}_{N}\right)$ since the ball and the polydisk are not biholomorphically isomorphic if $N>1$.

All above results about density can be expressed in the language of universality. If $X$ and $Y$ are topological spaces, a sequence $T_{n}: X \rightarrow Y(n \in \mathbb{N})$ of continuous mappings is called universal whenever the set $\mathcal{U}$ of elements $x \in X$ such that the orbit $\left\{T_{n} x\right\}_{1}^{\infty}$ is dense in $Y$ is not empty. Each element of $\mathcal{U}$ is said to be universal for $\left\{T_{n}\right\}_{1}^{\infty}$. If $X$ and $Y$ are topological vector spaces and each $T_{n}$ is linear then the words hypercyclic and universal are synonymous. See [12] and [13] for good updated surveys about these topics.

In the Theorem 2.3 of this note we prove that properly discontinuous action is necessary and sufficient for a sequence of automorphisms on $\mathbb{B}_{N}$ to generate a universal sequence of composition operators on $H^{p}\left(\mathbb{B}_{N}\right)$. In addition, the universality is even obtained in an algebraically generic way. Therefore, our statement extends or completes the above ones due to the aforementioned authors.

\section{Universality on $\boldsymbol{H}^{p}\left(\mathbb{B}_{N}\right)$}

Before establishing the main result of this section, we need the following assertion, which can be found in [11, Satz 1.2.2 and Satz 1.4.2], see also [12, Proposition 6].

Theorem 2.1 Let $X, Y$ be metrizable topological vector spaces with $X$ complete and $Y$ separable, and let $\Lambda=\left\{L_{n}\right\}_{1}^{\infty}$ be a sequence of continuous linear operators from $X$ to $Y$. Then the following statements are equivalent:

(a) The set of universal elements for $\Lambda$ is a residual subset of $X$.

(b) The set of universal elements for $\Lambda$ is a dense subset of $X$.

(c) The set $\left\{\left(x, L_{n}(x)\right): x \in X, n \in \mathbb{N}\right\}$ is dense in $X \times Y$.

If, in addition, there is a dense subset $C$ of $X$ such that $\lim _{n \rightarrow \infty} L_{n}(x)$ exists for all $x \in C$, then (a), (b) and (c) are equivalent to

(d) The set of universal elements for $\Lambda$ is not empty.

In addition, we will make use of the following result due to the first author (see [1, Theorem 2]), which tells us that, under adequate conditions, universality is an algebraically—not only topologically_generic property. Recall that a sequence $T_{n}: X \rightarrow Y(n \in \mathbb{N})$ of continuous linear mappings between two topological vector spaces is said to be densely hereditarily hypercyclic (DHHC) if, for each subsequence $\{n(1)<n(2)<\cdots\} \subset \mathbb{N}$, the sequence $\left\{T_{n(k)}\right\}_{1}^{\infty}$ has a dense set of hypercyclic vectors.

Theorem 2.2 Assume that $X, Y$ are two separable metrizable topological vector spaces. If $T_{n}: X \rightarrow Y(n \in \mathbb{N})$ is a DHHC sequence, then there is a dense linear submanifold $M$ in $X$ all of whose nonzero vectors are universal for $\left\{T_{n}\right\}_{1}^{\infty}$. 
We also need some background about Hardy spaces in the $N$-dimensional unit ball (see specially [8] and [15]). For $0<p<+\infty$ the Hardy space on $\mathbb{B}_{N}$ is defined [8, pages 20-26] as

$$
H^{p}\left(\mathbb{B}_{N}\right):=\left\{f \in H\left(\mathbb{B}_{N}\right):\|f\|_{p}:=\sup _{0<r<1}\left(\int_{\mathbb{S}_{N}}\left|f_{r}(\xi)\right|^{p} d \sigma_{N}(\xi)\right)^{1 / p}<+\infty\right\},
$$

where $f_{r}(\xi)=f(r \xi)(0<r<1)$ and $\sigma_{N}$ is the positive Borel measure on $\mathbb{S}_{N}:=$ [the unit sphere in $\left.\mathbb{C}^{N}\right]=\left\{z=\left(z_{1}, \ldots, z_{N}\right): \sum_{1}^{N}\left|z_{j}\right|^{2}=1\right\}$ that is rotation invariant (i.e. invariant under the unitary group) and normalized so that $\sigma_{N}\left(\mathbb{S}_{N}\right)=1$. If $p \geq 1$, $H^{p}\left(\mathbb{B}_{N}\right)$ is a Banach space for the norm $\|\cdot\|_{p}$. If $f \in H^{p}\left(\mathbb{B}_{N}\right)$, then the radial limit $f(\xi)=\lim _{r \rightarrow 1} f(r \xi)$ exists $\sigma_{N}$-almost everywhere and

$$
\|f\|_{p}=\left(\int_{\mathbb{S}_{N}}|f(\xi)|^{p} d \sigma_{N}(\xi)\right)^{1 / p}
$$

As in the one-dimensional case, convergence in this norm implies uniform convergence on compacta, and the set of polynomials in the variables $z_{1}, \ldots, z_{N}$ is dense in $H^{p}\left(\mathbb{B}_{N}\right)$.

In general, for $N>1$, a composition operator is not bounded as an operator from $H^{p}\left(\mathbb{B}_{N}\right)$ into itself. But in [9] it is proved that at least operators on $H^{p}\left(\mathbb{B}_{N}\right)$ induced by linear fractional maps-in particular, by automorphisms of $\mathbb{B}_{N}$-are bounded.

We are now ready to state the promised version of the Seidel-Walsh theorem for Hardy spaces on the $N$-dimensional ball.

Theorem 2.3 Let $\left\{S_{n}\right\}_{1}^{\infty} \subset \operatorname{Aut}\left(\mathbb{B}_{N}\right)$ and $p \in[1,+\infty)$. Consider the set

$$
\mathcal{U}=\left\{f \in H^{p}\left(\mathbb{B}_{N}\right):\left\{f \circ S_{n}: n \in \mathbb{N}\right\} \text { is dense in } H^{p}\left(\mathbb{B}_{N}\right)\right\} .
$$

Then the following properties are equivalent:

(a) $\mathcal{U}$ is residual in $H^{p}\left(\mathbb{B}_{N}\right)$.

(b) $\mathcal{U}$ is not empty.

(c) $\mathcal{U} \cup\{0\}$ contains a dense linear submanifold of $H^{p}\left(\mathbb{B}_{N}\right)$.

(d) The action of $\left\{S_{n}\right\}_{1}^{\infty}$ is properly discontinuous on $\mathbb{B}_{N}$.

Proof: That (a) implies (b) is trivial. If (b) holds then there exists $f \in H\left(\mathbb{B}_{N}\right)$ such that the orbit $\left\{f \circ S_{n}: n \in \mathbb{N}\right\}$ is dense in $H\left(\mathbb{B}_{N}\right)$, because convergence in $H^{p}\left(\mathbb{B}_{N}\right)$ implies compact convergence. Then (d) is satisfied by [14, Proposition 1].

For the part "(d) implies (a)", we will use Theorem 2.1. Recall that the set of polynomials is dense in $H^{p}\left(\mathbb{B}_{N}\right)$. Fix two polynomials $p(z), q(z)$ and a number $\varepsilon>0$. We should find a function $g \in H^{p}\left(\mathbb{B}_{N}\right)$ and a positive integer $n_{0}$ such that

$$
\|p-g\|_{p}<\varepsilon \text { and }\left\|q-\left(g \circ S_{n_{0}}\right)\right\|_{p}<\varepsilon .
$$


We first prove that there exists a point $\gamma \in \mathbb{S}_{N}$ such that, for some sequence $n(1)<$ $n(2)<\cdots<n(j)<\cdots$ of positive integers, $S_{n(j)}(z) \rightarrow \gamma(j \rightarrow \infty)$ for all $z \in \overline{\mathbb{B}_{N}}$ except for at most one point on $\mathbb{S}_{N}$. For this, note that for each $n \in \mathbb{N}, S_{n}=U_{n} \circ \varphi_{a_{n}}$, where $U_{n}$ is a unitary map and $a_{n} \in \mathbb{B}_{N}$. Since the action of $\left\{S_{n}\right\}_{1}^{\infty}$ is properly discontinuous, we have by [14, Proposition 2] that $\sup _{n \in \mathbb{N}}\left|a_{n}\right|=1$, so there exists a point $\gamma_{1} \in \mathbb{S}_{N}$ and a sequence $n(1)<n(2)<\cdots<n(j)<\cdots$ of positive integers such that $a_{n(j)} \rightarrow \gamma_{1}$ $(j \rightarrow \infty)$. Since very $U_{n}$ is unitary, $\left|U_{n}(z)\right|=|z|$ for all $z \in \mathbb{C}^{N}$ and all $n \in \mathbb{N}$; hence $\left\{U_{n}: n \in \mathbb{N}\right\}$ is uniformly bounded on compact sets in $\mathbb{C}^{N}$ and, by Montel's theorem and by taking a new subsequence if necessary, $\left\{U_{n(j)}\right\}_{1}^{\infty}$ converges to an entire function $U$, uniformly on $\overline{\mathbb{B}_{N}}$. Trivially, $|U(z)|=|z|$ for all $z \in \mathbb{C}^{N}$. Define $\gamma:=U\left(\gamma_{1}\right)$. Then $|\gamma|=\left|\gamma_{1}\right|=1$, that is, $\gamma \in \mathbb{S}_{N}$. Let us denote, for the sake of simplicity, $R_{j}=S_{n(j)}$ and $b_{j}=a_{n(j)}(j \in \mathbb{N})$. If $z=\left(z_{1}, \ldots, z_{n}\right) \in \mathbb{C}^{N}$ then $\bar{z}:=\left(\overline{z_{1}}, \ldots, \overline{z_{N}}\right)$. We now show that $R_{j}(z) \rightarrow \gamma(j \rightarrow \infty)$ for all $z \in \overline{\mathbb{B}_{N}} \backslash\left\{\overline{\gamma_{1}}\right\}$. We have that, for very $z \in \overline{\mathbb{B}_{N}}$,

$$
\begin{aligned}
\left|R_{j}(z)-\gamma\right| & =\left|\left(U_{n(j)} \circ \varphi_{b_{j}}\right)(z)-U\left(\gamma_{1}\right)\right| \\
& \leq\left|U_{n(j)}\left(\gamma_{1}\right)-U\left(\gamma_{1}\right)\right|+\left|U_{n(j)}\left(\varphi_{b_{j}}(z)\right)-U_{n(j)}\left(\gamma_{1}\right)\right| \\
& =\left|U_{n(j)}\left(\gamma_{1}\right)-U\left(\gamma_{1}\right)\right|+\left|\varphi_{b_{j}}(z)-\gamma_{1}\right|,
\end{aligned}
$$

because each $U_{n(j)}$ is unitary. Now, the first term of the last hand side tends to zero as $j \rightarrow \infty$. On the other hand,

$$
\begin{aligned}
& \left|\varphi_{b_{j}}(z)-\gamma_{1}\right| \\
& =\left|\frac{b_{j}-P_{b_{j}}(z)-\left(1-\left|b_{j}\right|^{2}\right)^{1 / 2} Q_{b_{j}}(z)}{1-<z, b_{j}>}-\gamma_{1}\right| \\
& \leq\left|\frac{b_{j}-\frac{<z, b_{j}>}{\left|b_{j}\right|^{2}} b_{j}}{1-<z, b_{j}>}-\gamma_{1}\right|+\left(1-\left|b_{j}\right|^{2}\right)^{1 / 2} \frac{\left|Q_{b_{j}}(z)\right|}{\left|1-<z, b_{j}>\right|} \\
& \leq\left|\frac{b_{j}-<z, b_{j}>b_{j}}{1-<z, b_{j}>}-\gamma_{1}\right|+\left|\frac{\frac{<z, b_{j}>}{\left|b_{j}\right|^{2}} b_{j}-<z, b_{j}>b_{j}}{1-<z, b_{j}>}\right|+\frac{\left(1-\left|b_{j}\right|^{2}\right)^{1 / 2}}{\mid 1-<z, b_{j}>1} \\
& \leq\left|b_{j}-\gamma_{1}\right|+\left(\frac{1}{\left|b_{j}\right|^{2}}+1\right) \frac{\left(1-\left|b_{j}\right|^{2}\right)^{1 / 2}}{\mid 1-<z, b_{j}>1} \rightarrow 0 \quad(j \rightarrow \infty)
\end{aligned}
$$

for all $z \in \overline{\mathbb{B}_{N}} \backslash\left\{\overline{\gamma_{1}}\right\}$, because $b_{j} \rightarrow \gamma_{1},\left|b_{j}\right|^{2} \rightarrow 1$ and $1-<z, b_{j}>\rightarrow 1-<z, \gamma_{1}>\neq 0$ $(j \rightarrow \infty)$. Therefore $S_{n(j)} \rightarrow \gamma(j \rightarrow \infty)$ pointwise on $\overline{\mathbb{B}_{N}} \backslash\left\{\overline{\gamma_{1}}\right\}$. Again with no loss of generality we can assume that $S_{n} \rightarrow \gamma(n \rightarrow \infty)$ on that set and, in particular, $S_{n}(z) \rightarrow \gamma$ $(n \rightarrow \infty)$ for $\sigma_{N}$-almost very point of $\mathbb{S}_{N}$.

Consider the peak function for $\gamma$ defined as

$$
a(z)=\frac{1+<z, \gamma>}{2},
$$


that is, $a(z)$ is continuous on $\overline{\mathbb{B}_{N}}$, holomorphic in $\mathbb{B}_{N}, a(\gamma)=1$ and $|a(z)|<1$ for all $z \in \overline{\mathbb{B}_{N}} \backslash\{\gamma\}$. Choose a positive integer $m$ such that

$$
\left\|a^{m}\right\|_{p}<\frac{\varepsilon}{\|p\|_{\infty}+\|q\|_{\infty}}
$$

which is possible because of the Lebesgue Bounded Convergence Theorem. Here $\|h\|_{\infty}:=$ $\max \left\{|h(z)|: z \in \overline{\mathbb{B}_{N}}\right\}$ for any continuous function $h$ on $\overline{\mathbb{B}_{N}}$. Since $a(z)$ is continuous and $a(\gamma)=1$, we have that

$$
1-\left[a\left(S_{n}(z)\right)\right]^{m} \rightarrow 0 \quad(n \rightarrow \infty)
$$

for $\sigma_{N}$-almost very point of $\mathbb{S}_{N}$. Again by the Lebesgue Bounded Convergence Theorem, we derive that $\left\|1-\left[a\left(S_{n}(\cdot)\right)\right]^{m}\right\|_{p} \rightarrow 0(n \rightarrow \infty)$, so there is $n_{0} \in \mathbb{N}$ with

$$
\left\|1-\left[a\left(S_{n_{0}}(\cdot)\right)\right]^{m}\right\|_{p}<\frac{\varepsilon}{\|p\|_{\infty}+\|q\|_{\infty}} .
$$

Let us define

$$
g(z)=p(z)+a(z)^{m}\left[q\left(S_{n_{0}}^{-1}(z)\right)-p(z)\right] .
$$

Then $g$ is continuous on $\overline{\mathbb{B}_{N}}$, so $g \in H^{p}\left(\mathbb{B}_{N}\right)$. We have, from (2.2) and (2.3), that

$$
\|p-g\|_{p}=\left\|a(z)^{m}\left[q\left(S_{n_{0}}^{-1}(z)\right)-p(z)\right]\right\|_{p}<\varepsilon
$$

and

$$
\begin{aligned}
& \left\|q(z)-\left(g \circ S_{n_{0}}\right)(z)\right\|_{p} \\
& \quad=\left\|q(z)-p\left(S_{n_{0}}(z)\right)-a\left(S_{n_{0}}(z)\right)^{m}\left[q(z)-p\left(S_{n_{0}}(z)\right)\right]\right\|_{p} \\
& \quad=\|\left(1-\left[a\left(S_{n_{0}}(z)\right)\right]^{m}\right)\left(q(z)-p\left(S_{n_{0}}(z)\right) \|_{p}<\varepsilon .\right.
\end{aligned}
$$

Consequently, (2.1) is satisfied. An application of Theorem 2.1 with $X=Y=$ $H^{p}\left(\mathbb{B}_{N}\right)$ and $L_{n}=C_{S_{n}}(n \in \mathbb{N})$ yields the desired result. Hence (a), (b) and (d) are equivalent.

Finally, it is evident that (c) implies (b). Thus, it is enough to show that (d) implies (c). Again by [14], we have that if (d) holds then $\sup _{n \in \mathbb{N}}\left|a_{n}\right|=1$. Therefore there exists a sequence $\{m(1)<m(2)<\cdots\} \subset \mathbb{N}$ with $\left|a_{m(j)}\right| \rightarrow 1$ as $j \rightarrow \infty$. But this yields $\left|a_{n(j)}\right| \rightarrow 1\left(\right.$ so $\sup _{n \in \mathbb{N}}\left|a_{n(j)}\right|=1$ ) for every subsequence $\{n(1)<n(2)<\cdots\} \subset$ $\{m(1)<m(2)<\cdots\}$. Once more, from [14] it is derived that the action of $\left\{C_{S_{n(j)}}\right\}_{1}^{\infty}$ is properly discontinuous, so each subsequence $\left\{C_{S_{n(j)}}\right\}_{1}^{\infty}$ of $\left\{C_{S_{m(j)}}\right\}_{1}^{\infty}$ has a dense set of hypercyclic vectors. Consequently, $\left\{C_{S_{m(j)}}\right\}_{1}^{\infty}$ is DHHC. By Theorem 2.2 (applied on $X=Y=H^{p}\left(\mathbb{B}_{N}\right)$ and $\left.T_{j}=C_{S_{m(j)}}\right)$, there exists a dense linear submanifold $M$ of $H^{p}\left(\mathbb{B}_{N}\right)$ such that $M \backslash\{0\}$ is included in the set $\mathcal{U}_{1}$ of universal vectors for $\left\{C_{S_{m(j)}}\right\}_{1}^{\infty}$. But, clearly, $\mathcal{U} \supset \mathcal{U}_{1}$. Hence $\mathcal{U} \supset M \backslash\{0\}$, and we are done.

In particular, Theorem 2.3 can be applied when $S_{n}$ is the $n$ th-iterate of a single $\varphi \in \operatorname{Aut}\left(\mathbb{B}_{N}\right)$ such that $\varphi$ has no fixed point in $\mathbb{B}_{N}$ because, in this case, the iterates of $\varphi$ 
tend uniformly on compacta to a point of the unit sphere (the "Denjoy-Wolff point" of $\varphi)$ : see [8, page 108]. As mentioned in Section 1, this last result-except for the algebraic genericity - has been obtained for the case $p=2$ by Xiaoman et al. [18] by using a rather different approach.

Acknowledgements. The first and third authors have been partially supported by the Plan Andaluz de Investigación de la Junta de Andalucía FQM-127 and by DGES Grant BFM2003-03893-C02-01. The second author has been partially supported by MEC and FEDER MTM2005-07347. The three authors have been partially supported by MEC (Acción Especial) MTM2004-21420-E.

\section{References}

[1] L. Bernal-González. Densely hereditarily hypercyclic sequences and large hypercyclic manifolds. Proc. Amer. Math. Soc., 127: 3279-3285, 1999.

[2] L. Bernal-González and A. Montes-Rodríguez. Universal functions for composition operators. Complex Variables, 27:47-56, 1995.

[3] C. D. Birkhoff. Démonstration d'un théorème élémentaire sur les fonctions entières. C. R. Acad. Sci. Paris, 189:473-475, 1929.

[4] A. Bonilla and M. C. Calderón-Moreno. On universality of composition operators in several variables. Houston Math. J., 27:907-918, 2001.

[5] P. S. Bourdon and J. H. Shapiro. Cyclic phenomena for Composition Operators. Memoirs of the Amer. Math. Soc., 596. Providence, Rhode Island, 1997.

[6] R. B. Burckel. Iterating analytic self-maps of discs. Amer. Math. Monthly, 88:396407, 1981.

[7] P. S. Chee. Universal functions in several complex variables. J. Austral. Math. Soc. (Series A), 28:189-196, 1979.

[8] C. C. Cowen and B. P. MacCluer. Composition operators on spaces of analytic functions. CRC Press, Boca Raton, 1995.

[9] C. C. Cowen and B. P. MacCluer. Linear fractional maps of the ball and their composition operators. Acta Sci. Math. (Szeged), 66:351-376, 2000.

[10] P. L. Duren. Theory of $H^{p}$ spaces, 2nd edition. Dover, Mineola, New York, 2000.

[11] K.-G. Grosse-Erdmann. Holomorphe Monster und universelle Funktionen. Mitt. Math. Sem. Giessen, 176, 1987.

[12] K.-G. Grosse-Erdmann. Universal families and hypercyclic operators. Bull. Amer. Math. Soc., 36:345-381, 1999.

[13] K.-G. Grosse-Erdmann. Recent developments in hypercyclicity. Rev. R. Acad. Cien. Serie A. Mat., 97:273-286, 2003. 
[14] F. León-Saavedra. Universal functions on the unit ball and the polydisk. Contemp. Math., 232:233-238, 1999.

[15] W. Rudin. Function Theory in the unit ball of $\mathbb{C}^{n}$. Springer-Verlag, New York, 1980.

[16] W. P. Seidel and J. L. Walsh. On approximation by uclidean and non-uclidean translates of an analytic function. Bull. Amer. Mat. Soc., 47:916-920, 1941.

[17] J. H. Shapiro. Composition operators and classical function theory. Springer-Verlag, New York, 1993.

[18] C. Xiaoman, C. Guangfu and G. Kunyu. Inner functions and cyclic composition operators on $H^{2}\left(B_{n}\right)$. J. Math. Anal. Appl., 250:660-669, 2000.

[19] K. Zhu. Operator Theory in Function Spaces. Marcel Dekker, New York, 1990.

L. Bernal-González

Departamento Análisis Matemático

Facultad de Matemáticas, apdo. 1160

Avd. Reina Mercedes

41080 Sevilla

Spain

lbernal@us.es

M. C. Calderón-Moreno

Departamento Análisis Matemático

Facultad de Matemáticas, apdo. 1160

Avd. Reina Mercedes

41080 Sevilla

Spain

mccm@us.es
A. Bonilla

Departamento Análisis Matemático

Universidad de La Laguna

Astrofísico Fco. Sánchez, s/n

38271 La Laguna

Spain

abonilla@ull.es 